\title{
Effect of Removable Partial Dentures on Masticatory Performance and Oral Health-related Quality of Life in Shortened Dental Arch Patients
}

\author{
Nay Nwe Htun ${ }^{1}$, Thiri Kyaw² ${ }^{2}$ Aung Thu Hein ${ }^{3}$, Kyaw Tint ${ }^{4}$, Shwe Hlaing ${ }^{5}$
}

\begin{abstract}
Aim and objective: This study aimed to evaluate the effect of removable partial dentures (RPDs) on masticatory performance (MP) and oral health-related quality of life (OHRQoL) in shortened dental arch (SDA) patients.

Materials and methods: Twenty mandibular SDA patients with four occlusal units, opposing with maxillary complete dentition, that were natural teeth or fixed prosthesis, were recruited and measured MP by using gummy jelly (Glucolumn, GC Co. Ltd., Tokyo, Japan) and Glucosensor GS-II (GC Co. Ltd., Tokyo, Japan) (glucose extraction method). In addition, OHRQoL was assessed by using the oral health impact profile-14 questionnaire, which consists of 14 questions with seven domains. The total score and individual domain scores were calculated and used as parameters of OHRQoL. At 2 weeks after the final adjustment of RPD, MP and OHRQoL were assessed by the same method and questionnaire. These data were compared before and after rehabilitation. The $p$ value of $<0.05$ was considered statistically significant.

Results: A total of 20 SDA patients consisting of 17 (85\%) females and $3(15 \%)$ males who met the prescribed criteria (45-68 years, mean; $56 \pm$ 8.23 years) were recruited. Both MP $(p<0.001)$ and OHRQoL $(p<0.001)$ were significantly improved after rehabilitation.

Conclusion: These results suggested that rehabilitation with RPDs significantly improves objective MP and subjective OHRQoL in patients with SDA.

Keywords: Glucose extraction method, Masticatory performance, Oral health-related quality of life, Rehabilitation, Removable partial denture, Shortened dental arch.

International Journal of Prosthodontics and Restorative Dentistry (2021): 10.5005/jp-journals-10019-1309
\end{abstract}

\section{INTRODUCTION}

By the year 2050, the percent of the elderly (60 years and over) is projected to more than double, with over 1.5 billion in the elderly population. ${ }^{1}$ Accompanying this, an increasing number of people are retaining their teeth into old age and becoming partial edentulism. ${ }^{2}$ In particular, posterior teeth are relatively at increased risk of loss, due to difficulty in performing efficient oral hygiene and the substantial amount of occlusal force in this area. ${ }^{3} \mathrm{~A}$ state of partial edentulism where the most distal teeth are missing is referred to as a shortened dental arch (SDA). ${ }^{4}$ Depending on the degree of shortening, SDA can be classified into four categories: (1) slightly SDA with 5-7 occlusal units (OUs), (2) moderately SDA with 3-4 OUs, (3) extremely SDA with 0-2 OUs, and (4) asymmetrical extremely SDA with no premolar occlusion on one side and more occluding pairs on the other side. ${ }^{5}$ An occluding pair of premolars means one OU, and an occluding pair of molars means two OUs. ${ }^{4}$ Possible implications of partial edentulism include masticatory inefficiency, changes in food selections, psychosocial problems, and decreased oral health-related quality of life (OHRQoL). ${ }^{6}$

One of the important goals of prosthodontic rehabilitation in partially edentulous patients is to restore masticatory function because the chewing ability is important both for oral health and the general health status. ${ }^{7}$ When tooth replacement is required for patients with SDA, cantilevered fixed prostheses, implant-supported fixed prostheses, implant-supported/retained removable partial dentures (RPDs), and conventional cast RPDs can be considered. Rehabilitation with RPDs is the most common option because it is relatively simple, non-invasive, and inexpensive while it
1,3,5 Department of Prosthodontics, University of Dental Medicine, Mandalay, Myanmar

${ }^{2,4}$ Department of Prosthodontics, University of Dental Medicine, Yangon, Myanmar

Corresponding Author: Nay Nwe Htun, Department of Prosthodontics, University of Dental Medicine, Mandalay, Myanmar, Phone: +959259277393, e-mail: naynwehtun3465@gmail.com

How to cite this article: Htun NN, Kyaw T, Hein AT, et al. Effect of Removable Partial Dentures on Masticatory Performance and Oral Health-related Quality of Life in Shortened Dental Arch Patients. Int J Prosthodont Restor Dent 2021;11(2):71-75.

Source of support: The Implementation Research Grant (2019-2020) of Ministry of Health and Sports, Myanmar (Project ID-172)

Conflict of interest: None

has high caries risk and periodontal destruction of abutment teeth (high biological cost). ${ }^{8}$ Therefore, another alternate option is the use of the SDA concept. In 1981, Kayser ${ }^{4}$ suggested that restoration of missing posterior teeth should be performed to the level of the functional dentition (second premolars) in older individuals, ideally in symmetric SDA patients with at least four OUs, because of their sufficient adaptive capacity to maintain adequate oral function. Therefore, the World Health Organization has proposed this concept as an adult oral health goal till the end of life. ${ }^{9}$ In addition, this concept is corroborated by observational studies related to occlusal stability, signs and symptoms of mandibular

\footnotetext{
() Jaypee Brothers Medical Publishers. 2021 Open Access This article is distributed under the terms of the Creative Commons Attribution 4.0 International License (https://creativecommons.org/licenses/by-nc/4.0/), which permits unrestricted use, distribution, and non-commercial reproduction in any medium, provided you give appropriate credit to the original author(s) and the source, provide a link to the Creative Commons license, and indicate if changes were made. The Creative Commons Public Domain Dedication waiver (http://creativecommons.org/publicdomain/zero/1.0/) applies to the data made available in this article, unless otherwise stated.
} 
dysfunction, oral function, oral comfort, and periodontal support in SDA patients. ${ }^{10}$ Nonetheless, this concept has been rejected by some studies on SDA in relation to temporomandibular joint dysfunction, ${ }^{11}$ increased risk to lose premolars, ${ }^{12}$ and brain activity. ${ }^{13}$

Not restoring SDA with distal extension RPD is due to the possible risk for abutment teeth deterioration, rather than providing clinically relevant posterior occlusal support, and perceived limited added value accounting for not wearing the RPD. ${ }^{14-16}$ On the other hand, it has been criticized because posterior teeth loss is related to a decrease in masticatory performance (MP), mandibular displacement, ${ }^{3}$ as well as various changes in the body. Masticatory inefficiency is believed to cause deficiencies in nutrition with various effects on general health and a preference for a more soft diet than vegetables. ${ }^{17,18}$ In addition, soft-diet feeding associated with masticatory deficiency due to tooth loss is a risk factor for the development of dementia and Alzheimer's disease. ${ }^{19}$ Thus, restoring SDA with RPD is to improve masticatory function and to re-establish the posterior occlusal support. ${ }^{20,21}$

Nevertheless, the SDA concept is still a controversial issue. In the case of a patient who seeks the restoration of lost molars, whether to replace or better not to replace is in doubt for most of the clinicians. Therefore, it was hypothesized that removable cast partial dentures for SDA patients improve MP and OHRQoL. This study aimed to evaluate the effect of distal extension RPDs on MP and OHRQoL in SDA patients.

\section{Materials and Methods}

This quasi-experimental study was carried out in the Department of Prosthodontics, between September 2019 and August 2020. A total of 20 mandibular partially edentulous patients were recruited consecutively according to the selection criteria as shown in Table 1. The sample size calculation was performed based on a previous study of similar nature in which a sample size with sufficient statistical power was achieved with 18 participants. ${ }^{22}$ Therefore, for statistical analysis and comparison for probable dropout, the total sample size was 20 (typical dropout percentage

Table 1: Inclusion and exclusion criteria

\begin{tabular}{l} 
Criteria \\
Inclusion criteria \\
- Age-45-70 years \\
- Gender-Both sexes \\
- Partially edentulous mandible: \\
- With 4 occlusal units (An occluding pair of premolars means \\
one occlusal unit, and an occluding pair of molars means \\
two occlusal units) \\
- Intact anterior region or restorable with conventional fixed \\
prostheses/implant-supported fixed prostheses \\
- Those opposing with complete maxillary dentition \\
$\quad$ prosthesis \\
Exclusion criteria \\
- Acute dental and periodontal diseases \\
Patients who had any signs or symptoms of \\
temporomandibular joint disorders \\
- Patients with mandibular torus \\
\hline
\end{tabular}

of $10 \%)$. This study was conducted after approval of protocol from the Board of Study (Prosthodontics) and the Research and Ethics Committee.

During the study period, informed consent was obtained from all patients participating in this study after a thorough explanation of the study procedure in accordance with the Declaration of Helsinki. Sociodemographic conditions such as age, sex, time of edentulism, and denture experience were collected at the time of evaluation. And then, MP and OHRQoL were assessed as a baseline evaluation.

All clinical and laboratory procedures were performed according to the standardized methods and principles used in the Department of Prosthodontics, in accordance with the standard operative procedure for infection control during the coronavirus disease 2019 pandemic. Each subject received conventional mandibular cast RPDs (Kennedy Class I or Class II) with artificial teeth up to second molars and the acrylic resin base extension up to the retromolar pad. At 2 weeks, after the final adjustment of RPD, MP, and OHRQoL were assessed by the same procedure. The data were recorded and compared between before rehabilitation and after rehabilitation.

Assessment of MP score was done by the glucose extraction method using cylindrical shaped gummy jelly (Glucolumn, GC Co. Ltd., Tokyo, Japan) and Glucosensor GS-II (GC Corporation, Tokyo, Japan). ${ }^{23,24}$ The validity, reliability, and reproducibility of this method have been reported previously. ${ }^{25,26}$ The subjects were requested to chew the gummy jelly on their habitual chewing side with all efforts for 20 seconds. Then, the participants were asked to take $10 \mathrm{~mL}$ of distilled water in their mouth, and to expectorate all the bolus and water into a filter cup from top of the mesh. Then, the mesh was immediately removed and the cup was lightly agitated for 10 seconds to make it uniform. Following this, the disposable collection brush was sufficiently impregnated with the filtrate and then it was dropped on a GS-II sensor chip. The concentration of the dissolved glucose $(\mathrm{mg} / \mathrm{dL})$ in the filtrate was considered as MP score. The glucose concentrations from three trials were averaged for each participant for statistical analyses.

To measure OHRQoL, oral health impact profile (OHIP-14) questionnaires were used. The OHIP-14 questionnaire has 14 items with seven domains derived from the OHIP-49 which is a 49 -item questionnaire. ${ }^{27}$ It has been previously validated. ${ }^{28,29}$ Subjects were asked how frequently they had encountered the impact of each item in the last month. Responses were made on a scale of 0 (never) to 4 (very often). Total scores and individual domain scores were calculated by summing all these scores from 14 questions without weighting. A total score of 56 is the highest and it means OHRQoL is the lowest. The lower the total OHIP scores, the better the OHRQoL outcomes are. The same questionnaires were administered by a research nurse before and after rehabilitation. In this study, the summary score of 14 items and individual domain scores were used as indices for OHRQoL.

Data entry and analysis were done by Statistical Package for Social Sciences (SPSS, v25.0; IBM Corp., Armonk, NY, USA). Normality was checked by the Kolmogorov-Smirnov test. As the data were normally distributed, a paired $t$-test was used for within-group comparisons. The effect size (ES) for the MP score and the OHIP summary score were calculated with the following function: (mean score after rehabilitation-mean score before rehabilitation)/ standard deviation (SD) of change score. The ES $<0.2$ is considered 
to be small, 0.4 is moderate, and $>0.8$ is large. ${ }^{30}$ The level of significance was set at a $p$ value of $<0.05$.

\section{Results}

A total of 20 mandibular SDA patients who met the prescribed criteria were recruited (45-68 years, mean age; $56 \pm 8.23$ years) during the study period. Of these subjects, $85 \%$ (17/20) were females and $15 \%(3 / 20)$ were males. The demographic characteristics of the patients were presented in Table 2. The MP after rehabilitation was significantly better than before rehabilitation (Table 3). The total and individual domain scores of OHIP-14 after rehabilitation were significantly lower than before rehabilitation (Table 4). The result of the within-subject comparison found large and statistically significant improvements in both MP $(\mathrm{ES}=1.22, p<0.001)$ and OHRQoL (ES $=-1.56, p<0.001)$ after rehabilitation with RPDs.

Table 2: Demographic characteristics of the subjects $(n=20)$

\begin{tabular}{lc}
\hline \multicolumn{2}{c}{ Demographic characteristics } \\
\hline Age (years) $^{\#}$ & $56(8.23)$ \\
Gender & \\
Male & $3(15)$ \\
Female & $17(85)$ \\
Time of edentulism (years) ${ }^{\#}$ & $10.34(10.78)$ \\
Denture experience & \\
$\quad$ Presence & $6(30)$ \\
Absence & $14(70)$ \\
\hline
\end{tabular}

\#mean (SD), \$n (\%)

Table 3: Mean values and standard deviations of masticatory performance before and after treatment $(n=20)$

\begin{tabular}{llll}
\hline $\begin{array}{l}\text { Masticatory performance }(\mathrm{mg} / \mathrm{dL}) \\
\text { Mean }(\mathrm{SD})\end{array}$ & & \\
\cline { 1 - 2 } $\begin{array}{l}\text { Before } \\
\text { rehabilitation }\end{array}$ & $\begin{array}{l}\text { After } \\
\text { rehabilitation }\end{array}$ & t statistics (df) & $p_{\text {-value }}{ }^{+}$ \\
\hline $130.80(26.78)$ & $177.48(38.76)$ & $-5.456(19)$ & $<0.001^{*}$ \\
\hline${ }^{\dagger}$ Paired $t$ test & & & \\
${ }^{*} p<0.001$ & & &
\end{tabular}

\section{Discussion}

The present study aimed to evaluate whether rehabilitation with RPDs in SDA patients could improve MP and OHRQoL. This study found that rehabilitation with RPD is an effective treatment option for mandibular SDA patients in terms of MP (objectively) and OHRQoL (subjectively).

The result of the within-subject comparison showed a statistically significant improvement in mean MP score from before rehabilitation to after rehabilitation $(p<0.001)$ indicating that rehabilitation with RPDs in mandibular SDA patients possibly improved objective MP by providing balanced occlusion. This result could be attributed to the presence of more functional tooth units to preserve the masticatory function after the replacement of missing posterior teeth. ${ }^{31}$ This finding is consistent with the previous studies, where treatment with RPD significantly improved MP. ${ }^{13,32-34}$ However, it is contrary to other studies where there was no significant difference in MP before and after rehabilitation with RPD. ${ }^{35,36}$ The variation in methods for assessing MP employed in these studies and the different study designs may be responsible for this discrepancy. For many years, Manly's sieving method has been used as a standard diagnostic method for MP, where a test food was chewed for a specified number of strokes, and then the bolus is retrieved from the oral cavity before measuring with sieves of various mesh sizes. However, this method is plagued with issues related to complicated manipulations and the time-consuming nature. Therefore, alternative straightforward strategies have been introduced utilizing silicone impression material, paraffin wax, chewing gum, or gummy jelly as the test foods. In the present study, the glucose extraction method was used because it is relatively simple and possible to standardize the properties of the gummy jelly as the test food. In addition, there are reports of positive correlations between the MP as measured by this method and that measured by the sieving method ${ }^{37}$ as well as the mixing ability test. ${ }^{26}$

The results of this study indicated that rehabilitation with RPDs in mandibular SDA patients significantly improved their OHRQoL. This finding is consistent with the previous studies ${ }^{22,38,39}$ where restoration of missing posterior teeth in SDA patients improved OHRQoL. In addition, within-subject comparison studies evaluating treatment outcomes before and after rehabilitation indicated that rehabilitation with RPDs improved OHRQoL, ${ }^{22,39}$

Table 4: Mean and standard deviations for total score and individual domain scores with 7 domains before and after rehabilitation $(n=20)$

\begin{tabular}{|c|c|c|c|c|}
\hline & \multicolumn{2}{|c|}{ OHIP score: Mean (SD) } & \multirow[b]{2}{*}{ t statistics (df) } & \multirow[b]{2}{*}{ pvalue $e^{t}$} \\
\hline & $\begin{array}{l}\text { Before } \\
\text { rehabilitation }\end{array}$ & After rehabilitation & & \\
\hline Total score of OHIP-14 & $15.85(8.96)$ & $4.20(4.21)$ & $6.993(19)$ & $<0.001^{* * *}$ \\
\hline \multicolumn{5}{|l|}{ Domain } \\
\hline - Functional limitation & $0.80(1.40)$ & $0.35(0.67)$ & $1.484(19)$ & 0.154 \\
\hline - Pain & $4.00(1.75)$ & $1.60(1.43)$ & $4.857(19)$ & $<0.001^{* * *}$ \\
\hline - Psychological discomfort & $2.45(2.09)$ & $0.90(1.02)$ & $3.538(19)$ & $0.002^{* *}$ \\
\hline - Physical disability & $3.45(2.24)$ & $0.30(0.92)$ & $7.416(19)$ & $<0.001^{* * *}$ \\
\hline - Psychological disability & $1.90(2.05)$ & $0.70(1.26)$ & $3.335(19)$ & $0.003^{* *}$ \\
\hline - Social disability & $1.75(1.71)$ & $0.10(0.31)$ & $4.714(19)$ & $<0.001^{* * *}$ \\
\hline - Handicap & $1.50(1.54)$ & $0.25(0.64)$ & $3.526(19)$ & $0.002^{* *}$ \\
\hline
\end{tabular}

${ }^{\dagger}$ Paired $t$ test

${ }^{* *} p<0.01,{ }^{* * *} p<0.001$ 
while between-subject comparison studies found that RPDs in SDA patients did not indicate more statistically significant improvement in OHRQoL than the treatment according to the SDA concept. ${ }^{38,40-42}$ A possible explanation for these results may be the fact that only patients suffering from decreased quality of life sought prosthodontic treatments to fulfill their needs. ${ }^{20,22}$

With regard to individual domains of the OHIP-14, the present study found that there were statistically significant reductions in mean scores of all domains except functional limitation, despite a reduction from before rehabilitation ( 0.80 points) to after rehabilitation ( 0.35 points). This can be explained by the fact that the subjects have the lowest impact on functional limitation domains before rehabilitation among seven domains.

Of all the OHIP domains, the pain had the highest mean score in all participants before rehabilitation with RPDs. Among two questions regarding physical pain, patients scored impact mainly in the question concerned with "uncomfortable to eat" at pretreatment. This may be due to masticatory load and pain on mastication acting on remaining alveolar ridge and masticatory mucosa in the areas of missing posterior teeth. Therefore, difficult to eat comfortably might be the reason why they sought treatments in SDA patients.

Some researchers analyzed the effect of RPDs on OHRQoL in SDA patients using the OHIP-49. Wolfart et al. found significant improvements in all OHIP domains. ${ }^{38}$ However, Fueki et al. found that significant improvements were observed in functional limitation domain and psychological discomfort domain. ${ }^{22}$ In addition, Omo et al. reported that there were significant improvements in all domains of OHIP-49 except functional limitation and handicap domains after rehabilitation with mandibular RPDs. ${ }^{39}$

Moreover, the ES for MP and OHIP summary scores were estimated for benchmarks of the effect of rehabilitation with RPDs in mandibular SDA patients in this study. As the ES for both MP and OHIP summary scores were $>0.8$, there were large and clinically significant improvements in MP and OHRQoL after rehabilitation with RPDs. Furthermore, in terms of clinical significance, whether the treatment effect on patient perceptions is more than a minimally important difference (MID) should be considered. The concept of MID for OHRQoL has been demonstrated as the smallest score difference that subjects perceive as being advantageous. ${ }^{43,44}$ Despite being not constant in various settings, to assess clinical significance in terms of patient-based outcomes, the MID in OHIP-14 summary scores (a 5-point change in OHIP-14 summary score) from previous studies was used as a norm. ${ }^{45,46} \mathrm{~A}$ recent study proposed that a 3-point change in OHIP-14 summary score was clinically meaningful in RPD treatments. ${ }^{47}$ In the present study, the mean change in summary score (11.65 points) is substantially larger than the proposed MID indicating that rehabilitation with RPDs in mandibular SDA patients is not only statistically significant but also clinically significant. Therefore, the results of this study suggest that rehabilitation with RPDs offers not only statistically but also a clinically significant improvement in OHRQoL in mandibular SDA patients who sought prosthetic treatments.

Although this study was designed to investigate the effect of RPD treatment both on objective MP and subjective OHRQoL in mandibular SDA patients, it has some limitations such as a small sample size with a larger participation of women and limited follow-up interval. Thus, a study with a larger sample size with equal distribution of sex and a longer follow-up period is needed to validate the conclusions of this study. A variety of positive outcomes such as nutritional status, cost-effectiveness, the longevity of RPD, and maintenance of the health of remaining oral tissue should be considered in clinical decision-making.

\section{CONCLUSION}

Within the limitations of this study, rehabilitation with RPDs significantly improves objective MP and subjective OHRQoL in mandibular SDA patients.

\section{ACKNOWLedgments}

This work was partly funded by the Implementation Research Grant (2019-2020) of Ministry of Health and Sports (Project ID-172). This study was conducted after approval of protocol from the Board of Study (Prosthodontics) and the Research and Ethics Committee of the University.

\section{References}

1. United Nations. Department of Economic and Social Affairs. Population Division. World population ageing, 2019 highlights. New York: United Nations, 2019.

2. Steele J, O'Sullivan I, Executive Summary: Adult Dental Health Survey 2009. The Health and Social Care Information Centre, 2011.

3. Iwashita $\mathrm{H}$, Tsukiyama $\mathrm{Y}$, Kori $\mathrm{H}$, et al. Comparative cross-sectional study of masticatory performance and mastication predominance for patients with missing posterior teeth. J Prosthodont Res 2014;58(4):223-229. DOI: 10.1016/j.jpor.2014.04.002.

4. Kayser AF. Shortened dental arches and oral function. J Oral Rehabil 1981;8(5):457-462. DOI: 10.1111/j.1365-2842.1981.tb00519.x.

5. Witter DJ, Hoefnagel RA, Snoek PA, et al. Extension of (extremely) shortened dental arches by fixed or removable partial dentures. Ned Tijdschr Tandheelkd 2009;116(11):609-614.

6. McLister C, Donnelly M, Cardwell CR, et al. Effectiveness of prosthodontic interventions and survival of remaining teeth in adult patients with shortened dental arches-a systematic review. J Dent 2018;78:31-39. DOI: 10.1016/j.jdent.2018.02.003.

7. Locker D, Matear D, Stephens M, et al. Oral health-related quality of life of a population of medically compromised elderly people. Community Dent Health 2002;19(2):90-97.

8. MacEntee MI. Biologic sequelae of tooth replacement with removable partial dentures: a case for caution. J Prosthet Dent 1993;70(2):132134. DOI: 10.1016/0022-3913(93)90007-B.

9. WHO Expert Committee on Recent Advances in Oral Health. Recent advances in oral health: report of a WHO Expert Committee. Geneva: World Health Organization; 1992. pp. 16-17.

10. Kanno T, Carlsson GE. A review of the shortened dental arch concept focusing on the work by the Käyser/Nijmegen group. J Oral Rehabil 2006;33(11):850-862. DOI: 10.1111/j.1365-2842.2006.01625.x.

11. Wang MQ, Xue F, He J, et al. Missing posterior teeth and risk of temporomandibular disorders. J Dent Res 2009;88(10):942-945. DOI: 10.1177/0022034509344387.

12. Gerritsen AE, Witter DJ, Bronkhorst EM, et al. Increased risk for premolar tooth loss in shortened dental arches. J Dent 2013;41(8):72 6-731. DOI: 10.1016/j.jdent.2013.05.013.

13. Shoi K, Fueki K, Usui N, et al. Influence of posterior dental arch length on brain activity during chewing in patients with mandibular distal extension removable partial dentures. J Oral Rehabil 2014;41(7): 486-495. DOI: 10.1111/joor.12169.

14. Preshaw PM, Walls AWG, Jakubovics NS, et al. Association of removable partial denture use with oral and systemic health. J Dent 2011;39(11):711-719. DOI: 10.1016/j.jdent.2011.08.018.

15. Creugers NHJ, Witter DJ, Van't Spijker A, et al. Occlusion and temporomandibular function among subjects with mandibular distal extension removable partial dentures. Int J Dent 2010;2010:1-7. DOI: $10.1155 / 2010 / 807850$. 
16. Graham R, Mihaylov S, Jepson N, et al. Determining "need" for a removable partial denture: a qualitative study of factors that influence dentist provision and patient use. Br Dent J 2006;200(3):155158. DOI: $10.1038 /$ sj.bdj.4813193.

17. Krall E, Hayes C, Garcia R. How dentition status and masticatory function affect nutrient intake. J Am Dent Assoc 1998;129(9):12611269. DOI: 10.14219/jada.archive.1998.0423.

18. Yoshida M, Kikutani T, Yoshikawa M, et al. Correlation between dental and nutritional status in community-dwelling elderly Japanese. Geriatr Gerontol Int 2011;11(3):315-319. DOI: 10.1111/j.14470594.2010.00688.x.

19. De Cicco V, Barresi M, Fantozzi MPT, et al. Oral implant-prostheses: new teeth for a brighter brain. PLoS ONE 2016;11(2):e0148715. DOI: 10.1371/journal.pone.0148715.

20. Fueki $K$, Igarashi $Y$, Maeda $Y$, et al. Factors related to prosthetic restoration in patients with shortened dental arches: a multicentre study. J Oral Rehabil 2010;38(7):525-532. DOI: 10.1111/j.13652842.2010.02183.x.

21. Kondo T, Wakabayashi N. Influence of molar support loss on stress and strain in premolar periodontium: a patient-specific FEM study. J Dent 2009;37(7):541-548. DOI: 10.1016/j.jdent.2009.03. 015.

22. Fueki K, Igarashi Y, Maeda Y, et al. Effect of prosthetic restoration on oral health-related quality of life in patients with shortened dental arches: a multicentre study. J Oral Rehabil 2015;42(9):701-708. DOI: 10.1111/joor.12297.

23. Morita $\mathrm{K}$, Tsuka $\mathrm{H}$, Kato $\mathrm{K}$, et al. Factors related to masticatory performance in healthy elderly individuals. J Prosthodont Res 2018;62(4):432-435. DOI: 10.1016/j.jpor.2018.03.007.

24. Takeshima T, Fujita Y, Maki K. Factors associated with masticatory performance and swallowing threshold according to dental formula development. Arch Oral Biol 2019;99:51-57. DOI: 10.1016/j. archoralbio.2018.12.012.

25. Shiga H, Kobayashi Y, Arakawa I, et al. Validation of a portable blood glucose testing device in measuring masticatory performance. Prosthodont Res Pract 2006;5(1):15-20. DOI: 10.2186/prp.5.15.

26. Sugiura T, Fueki K, Igarashi Y. Comparisons between a mixing ability test and masticatory performance tests using a brittle or an elastic test food. J Oral Rehabil 2009;36(3):159-167. DOI: 10.1111/j.13652842.2008.01917.x.

27. Slade GD. Derivation and validation of a short-form oral health impact profile. Community Dent Oral Epidemiol 1997;25(4):284-290. DOI: 10.1111/j.1600-0528.1997.tb00941.x.

28. Soe KK, Gelbier S, Robinson PG. Reliability and validity of two oral health related quality of life measures in Myanmar adolescents. Community Dent Health 2004;21(4):139-144.

29. Nyan $M, O o S Z$, Hlaing EE, et al. A study of relationship between partial edentulism and oral health related quality of life (OHRQoL) and effect of removable partial dneutre treatment on OHRQoL. Myanmar Dent J 2019;26(1):41-46.

30. Cohen J. Statistical power analysis for the behavioural science. 2nd ed., Lawrence Erlbaum Associates; 1988. pp. 8-13.

31. Yamashita S, Sakai S, Hatch JP, et al. Relationship between oral function and occlusal support in denture wearers. J Oral Rehabil 2000;27(10):881-886. DOI: 10.1046/j.1365-2842.2000. 00602.x.
32. Arce-Tumbay J, Sanchez-Ayala A, Sotto-Maior B, et al. Mastication in subjects with extremely shortened dental arches rehabilitated with removable partial dentures. Int J Prosthodont 2011;24(6):517-519.

33. Fueki K, Igarashi $Y$, Maeda $Y$, et al. Effect of prosthetic restoration on masticatory function in patients with shortened dental arches: a multicentre study. J Oral Rehabil 2016;43(7):534-542. DOI: 10.1111/ joor.12387.

34. Omo J, Sede M, Esan T. Masticatory efficiency of shortened dental arch subjects with removable partial denture: a comparative study. Niger J Clin Pract 2017;20(4):459-463. DOI: 10.4103/1119-3077.181363.

35. Aras K, Hasanreisoglu U, Shinogaya T. Masticatory performance, maximum occlusal force, and occlusal contact area in patients with bilaterally missing molars and distal extension removable partial dentures. Int J Prosthodont 2009;22(2):204-209.

36. Sánchez-Ayala A, Gonçalves TMSV, Ambrosano GMB, et al. Influence of length of occlusal support on masticatory function of free-end removable partial dentures: short-term adaptation. J Prosthodont 2013;22(4):313-318. DOI: 10.1111/j.1532-849X.2012.00938.x.

37. Kobayashi Y, Shiga H, Arakawa I, et al. The effectiveness of measuring glucose extraction for estimating masticatory performance. Prosthodont Res Pract 2006;5(2):104-108. DOI: 10.2186/prp.5.104.

38. Wolfart S, Müller F, Gerß J, et al. The randomized shortened dental arch study: oral health-related quality of life. Clin Oral Investig 2014;18(2):525-533. DOI: 10.1007/s00784-013-0991-6.

39. Omo J, Sede M, Esan T. Quality of life in subjects with shortened dental arch rehabilitated with removable metal-based partial dentures. Eur J Prosthodont 2016;4(2):25-31. DOI: 10.4103/2347-4610.190606.

40. McKenna G, Allen F, Woods N, et al. Cost-effectiveness of tooth replacement strategies for partially dentate elderly: a randomized controlled clinical trial. Community Dent Oral Epidemiol 2014;42(4):366-374. DOI: 10.1111/cdoe.12085.

41. McKenna G, Allen PF, O'Mahony D, et al. The impact of rehabilitation using removable partial dentures and functionally orientated treatment on oral health-related quality of life: a randomised controlled clinical trial. J Dent 2015;43(1):66-71. DOI: 10.1016/j. jdent.2014.06.006.

42. Reissmann DR, Wolfart S, John MT, et al. Impact of shortened dental arch on oral health-related quality of life over a period of 10 years a randomized controlled trial. J Dent 2019;80:55-62. DOI: 10.1016/j. jdent.2018.10.006.

43. Jaeschke R, Singer J, Guyatt GH. Measurement of health status. Ascertaining the minimal clinically important difference. Control Clin Trials 1989;10(4):407-415. DOI: 10.1016/0197-2456(89)90005-6.

44. Tsakos G, Allen PF, Steele JG, et al. Interpreting oral health-related quality of life data. Community Dent Oral Epidemiol 2012;40(3):193200. DOI: 10.1111/j.1600-0528.2011.00651.x.

45. Locker D, Jokovic A, Clarke M. Assessing the responsiveness of measures of oral health-related quality of life. Community Dent Oral Epidemiol 2004;32(1):10-18. DOI: 10.1111/j.1600-0528.2004.00114.x.

46. Tan H, Peres KG, Peres MA. Retention of teeth and oral healthrelated quality of life. J Dent Res 2016;95(12):1350-1357. DOI: 10.1177/0022034516657992.

47. Myint Oo KZ, Fueki K, Yoshida-Kohno E, et al. Minimal clinically important differences of oral health-related quality of life after removable partial denture treatments. J Dent 2020;92:103246. DOI: 10.1016/j.jdent.2019.103246. 